\title{
Safe Intravenous Thrombolysis after Traumatic Cardiopulmonary Resuscitation with Rib Fractures: A Case Report
}

\author{
Andrea Dressing Zina Graeter Juergen Bardutzky \\ Department of Neurology and Clinical Neuroscience, University Medical Center Freiburg, \\ Freiburg, Germany
}

\section{Keywords}

Intravenous thrombolysis - Acute stroke - Cardiopulmonary resuscitation - Rib fracture .

Case report

\begin{abstract}
We report a case of successful intravenous thrombolysis for a distal middle cerebral artery occlusion shortly after traumatic cardiopulmonary resuscitation due to an episode of ventricular tachycardia. A high prevalence of fatal cardiac arrhythmias in acute stroke patients raises the question of safety when administrating thrombolytic therapy after traumatic cardiopulmonary resuscitation; guidelines do not provide a satisfactory statement about this. Our case suggests that intravenous tissue-type plasminogen activator for acute ischemic stroke can be administered after a thorough risk-to-benefit evaluation without major adverse effects in patients after traumatic cardiopulmonary resuscitation, as bleeding complications seem rare and can be monitored and treated.

(C) 2017 The Author(s)

Published by S. Karger AG, Basel
\end{abstract}

\section{Introduction}

Intravenous thrombolysis (IVT) with recombinant tissue-type plasminogen activator (rt-PA) within $4.5 \mathrm{~h}$ after onset is a well-established and effective treatment for patients with 
acute ischemic stroke [1]. In critical care stroke medicine, the question arises whether rt-PA can be safely administered in a dosage conforming with the guidelines after traumatic cardiopulmonary resuscitation (CPR). The high prevalence of cardiac arrhythmias after stroke leads to a significant risk of malignant cardiac arrhythmias, such as ventricular tachycardia or ventricular fibrillation, and the need for CPR in the pre-hospital or hospital phase of treatment in acute stroke patients [2,3].

American and European guidelines include indications and criteria for the eligibility of patients and provide a list of absolute and relative contraindications [4, 5]. However, traumatic CPR with consequential rib fractures is not mentioned as a contraindication in the guidelines. Only trauma within 3 months prior to stroke is listed as a relative contraindication for the administration of rt-PA; however, both guidelines note that, after carefully considering the risks and benefits, patients may receive fibrinolytic therapy despite the presence of one or more relative contraindications.

The literature provides no evidence for the safety when administrating rt-PA after traumatic CPR with rib fractures - which can be regarded as acute trauma -, and case reports are sparse. In the literature, only one case report of a stroke patient receiving IVT after spontaneous, initially unrecognized rib fractures has been published [6]. To support future risk-to-benefit evaluations, we report a case of IVT after traumatic CPR with rib fractures without the occurrence of major bleeding complications.

\section{Case Presentation}

A 93-year-old male was admitted to our stroke unit with sudden-onset multimodal neglect, left-sided hemiparesis, and dysarthria. His National Institute of Health Stroke Scale (NIHSS) score on admission was 13. His vital signs on admission were stable. His past medical history included coronary heart disease with myocardial infarction and coronary stent implantation in 2006 and atrial fibrillation without the need for oral anticoagulants. He was regularly taking acetylsalicylic acid. Furthermore, he was diagnosed with arterial hypertension and a melanoma in situ on his forehead.

Shortly after admission, the patient lost consciousness due to ventricular tachycardia without a pulse. CPR was performed and the ventricular tachycardia was self-limiting after $30 \mathrm{~s}$ without the need for defibrillation. After that, the patient was stable to undergo imaging diagnostics. His high-sensitive troponin levels were initially raised $(0.031 \mathrm{ng} / \mathrm{dL})$, most likely due to an increased serum creatinine level (1.54 mg/dL) [7].

A multimodal computed tomography (CT) study, including noncontrast CT, CTangiography, and CT-perfusion, showed an area of restricted perfusion in the right parietal area of the middle cerebral artery territory without signs of intracranial hemorrhage (Fig. 1); no proximal artery occlusion could be detected. An additional CT scan of the thorax detected acute rib fractures of the 7th and 8th rib on the left side (Fig. 2), potentially as a consequence of the traumatic CPR. Due to the patient's severely disabling symptoms with an NIHSS score of 13 , we initiated therapy with body-weight-adjusted rt-PA $(0.9 \mathrm{mg} / \mathrm{kg}$ body weight $)$ with an onset-to-needle time of $135 \mathrm{~min}$ and a door-to-needle time of $75 \mathrm{~min}$; rt-PA was administered $60 \mathrm{~min}$ after CPR. Thrombectomy was not possible because of the distal localization of the artery occlusion. Therapy was performed with consent of the patient's next of kin.

Following IVT with an increased risk of bleeding, the patient was monitored in our intensive care unit. He developed a cutaneous hematoma on the right side of the chest, distant from the fractures. Chest radiographs (on days 5 and 6 after IVT) did not reveal any signs of 


\section{Case Reports in Neurology}

Dressing et al.: Safe Intravenous Thrombolysis after Traumatic Cardiopulmonary Resuscitation with Rib Fractures: A Case Report

bleeding complication (such as subpleural or chest wall hematomas or hemothorax), the patient showed cardiopulmonary stability. His blood tests revealed slightly decreasing hemoglobin levels (12.7 g/dL on admission, $9.6 \mathrm{~g} / \mathrm{dL}$ on day 1 after IVT, $7.8 \mathrm{~g} / \mathrm{dL}$ at discharge), which were most likely due to the cutaneous hematoma and the administration of intravenous fluids. His troponin levels remained stable. The cardiological diagnostics (ECG and transthoracic echocardiography) revealed no pathologies. Coronary angiography was postponed. The patient had a significant benefit from IVT and made a favorable recovery within 1 day (NIHSS score of 2 at discharge). The CT scan $24 \mathrm{~h}$ after symptom onset did not show any new infarctions in the territory of the right middle cerebral artery. He was discharged 10 days after admission.

\section{Discussion}

To our knowledge, this is the first report of a patient successfully treated with IVT after traumatic CPR and acute rib fractures without bleeding complications. In the literature, only one case report of a patient receiving IVT after spontaneous rib fractures has been published, where bleeding complications occurred and were treated successfully by placing chest tubes [6]. It is important to understand the circumstances under which rt-PA can be administered safely in order to evaluate the risks and benefits in patients with acute stroke, to look for the presence of relative contraindications, and to avoid delaying or withholding of an effective treatment option in as many cases as possible without endangering patients. In our case, IVT was unfortunately administered with delay, due to difficulties in decision making.

In general, there are 3 important points to consider in patients presenting with an indication for IVT after acute CPR with rib fractures:

- First, the question whether CPR with acute rib fractures should be regarded as a trauma - and, thus, should be classified as a relative contraindication - has not been answered in the guidelines or in the literature. This decision - whether to treat patients after traumatic CPR with rt-PA or not - must be made on an individual basis. Studies in patients with acute lung artery embolism and CPR demonstrated that serious bleeding complications rarely occur when these patients were treated with rt-PA [8, 9], but these complications can be dealt with. Although traumatic CPR with rib fractures is not listed explicitly among the relative contraindications, it increases the risk of bleeding complications, and, thus, should be considered in a risk-to-benefit evaluation. In the present case, we thought that the disabling deficits with an NIHSS score of 13 justified the therapy with rt-PA.

- Second, in cases with cardiac arrest, the underlying condition should be included in the therapeutic decision-making process regarding treatment with IVT. After acute myocardial infarction, a further relative contraindication is given [4, 5]. In our case, cardiac arrhythmia due to myocardial infarction seemed unlikely, supporting our decision to treat with rt-PA.

- Third, an effort should be made to diagnose consequences of trauma of CPR. In our case, the patient developed a subcutaneous hematoma, which has to be regarded as a possible complication of IVT, though it was located distantly from the rib fractures. Fortunately, our patient did not suffer from any major adverse events after IVT.

After IVT and in the presence of a previous trauma (traumatic rib fractures in our case), it is most important to monitor thoroughly for vital bleeding complications, such as bleeding into the thoracic cavity and hemothorax, by CT or radiographic imaging and regular blood 
tests. Potential bleeding complications can be treated by placing a chest tube and providing blood transfusions, and these treatment options must be provided.

\section{Conclusion}

CPR with acute rib fractures might present a relative contraindication for IVT, as it contains a higher risk of hemorrhagic complications; however, a study on a large cohort of patients treated with IVT after acute (rib) fractures is necessary to sufficiently answer this question. Potential bleeding complications can be successfully treated, if they are recognized early. Thus, in our opinion, after carefully balancing the risks and benefits, IVT may be considered in patients with traumatic CPR, keeping the potential complications and their treatments in mind. Traumatic CPR should not generally exclude patients from beneficial treatment with IVT.

\section{Statement of Ethics}

The authors have no ethical conflicts to declare.

\section{Disclosure Statement}

The authors have no conflicts of interest to declare.

\section{References}

1 Lees KR, von Kummer R, Brott TG, et al: Time to treatment with intravenous alteplase and outcome in stroke: an updated pooled analysis of ECASS, ATLANTIS, NINDS, and EPITHET trials. Lancet 2010;375:1695-1703.

-2 Kallmünzer B, Breuer L, Kahl N, et al: Serious cardiac arrhythmias after stroke: incidence, time course, and predictors - a systematic, prospective analysis. Stroke 2012;43:2892-2897.

-3 Ruthirago D, Julayanont P, Tantrachoti P, Kim J, Nugent K: Cardiac arrhythmias and abnormal electrocardiograms after acute stroke. Am J Med Sci 2016;351:112-118.

-4 European Stroke Organisation (ESO) Executive Committee, and ESO Writing Committee: Guidelines for management of ischaemic stroke and transient ischaemic attack 2008. Cerebrovasc Dis 2008;25:457507.

-5 Jauch EC, Saver JL, Adams HP Jr, et al: Guidelines for the early management of patients with acute ischemic stroke: a guideline for healthcare professionals from the American Heart Association/American Stroke Association. Stroke 2013;44:870-947.

-6 Shirokane K, Umeoka K, Mishina M, Mizunari T, Kobayashi S, Teramoto A: Hemothorax after the intravenous administration of tissue plasminogen activator in a patient with acute ischemic stroke and rib fractures. J Nippon Med Sch 2014;81:43-47.

7 Stacy SR, Suarez-Cuervo C, Berger Z, Wilson LM, Yeh HC, Bass EB, Michos ED: Role of troponin in patients with chronic kidney disease and suspected acute coronary syndrome: a systematic review. Ann Intern Med 2014;161:502-512.

-8 Janata K, Holzer M, Kürkciyan I, et al: Major bleeding complications in cardiopulmonary resuscitation: the place of thrombolytic therapy in cardiac arrest due to massive pulmonary embolism. Resuscitation 2003;57:49-55.

-9 Lederer W, Lichtenberger C, Pechlaner C, Kroesen G, Baubin M: Recombinant tissue plasminogen activator during cardiopulmonary resuscitation in 108 patients with out-of-hospital cardiac arrest. Resuscitation 2001;50:71-76. 


\section{Case Reports in Neurology}

\begin{tabular}{l|l}
\hline Case Rep Neurol 2017;9:156-160 \\
\hline DOI: 10.1159/000477392 & $\begin{array}{l}\text { C 2017 The Author(s). Published by S. Karger AG, Basel } \\
\text { www.karger.com/crn }\end{array}$ \\
\hline
\end{tabular}

Dressing et al.: Safe Intravenous Thrombolysis after Traumatic Cardiopulmonary Resuscitation with Rib Fractures: A Case Report
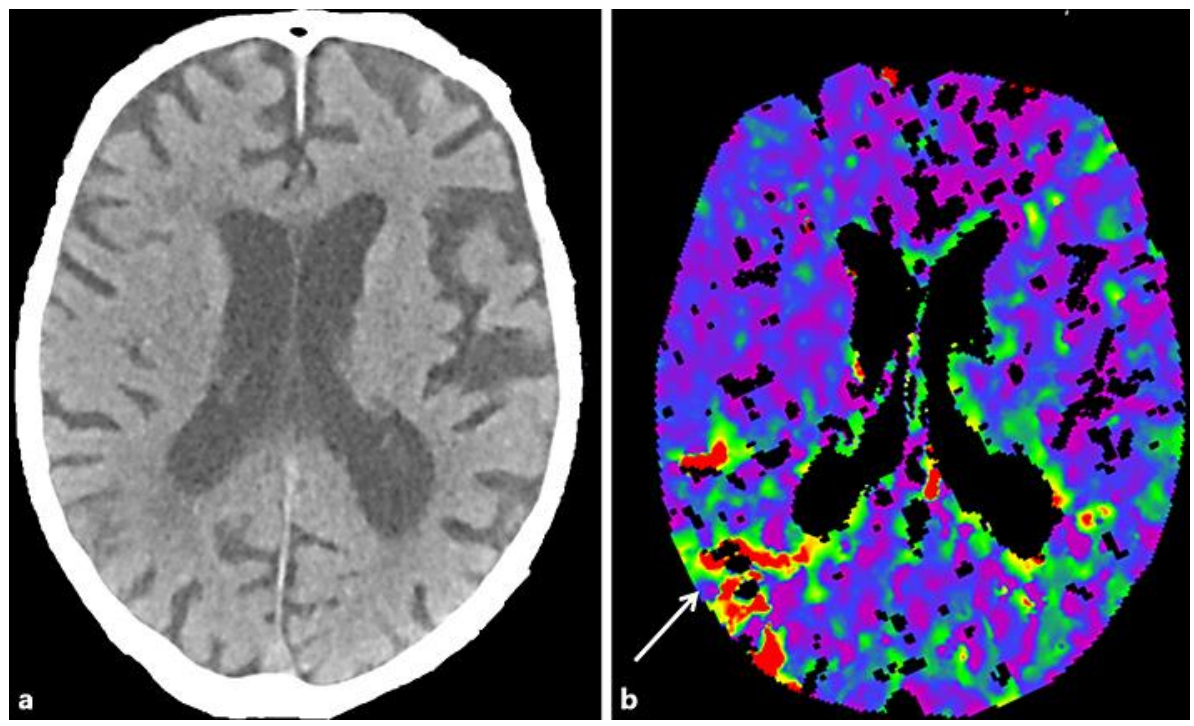

Fig. 1. Multimodal CT imaging shows no early signs of infarction (a), but an area of restricted perfusion (Tmax) in the right parietal lobe in the middle cerebral artery territory (indicated by an arrow; b). Color bar indicates Tmax in seconds.
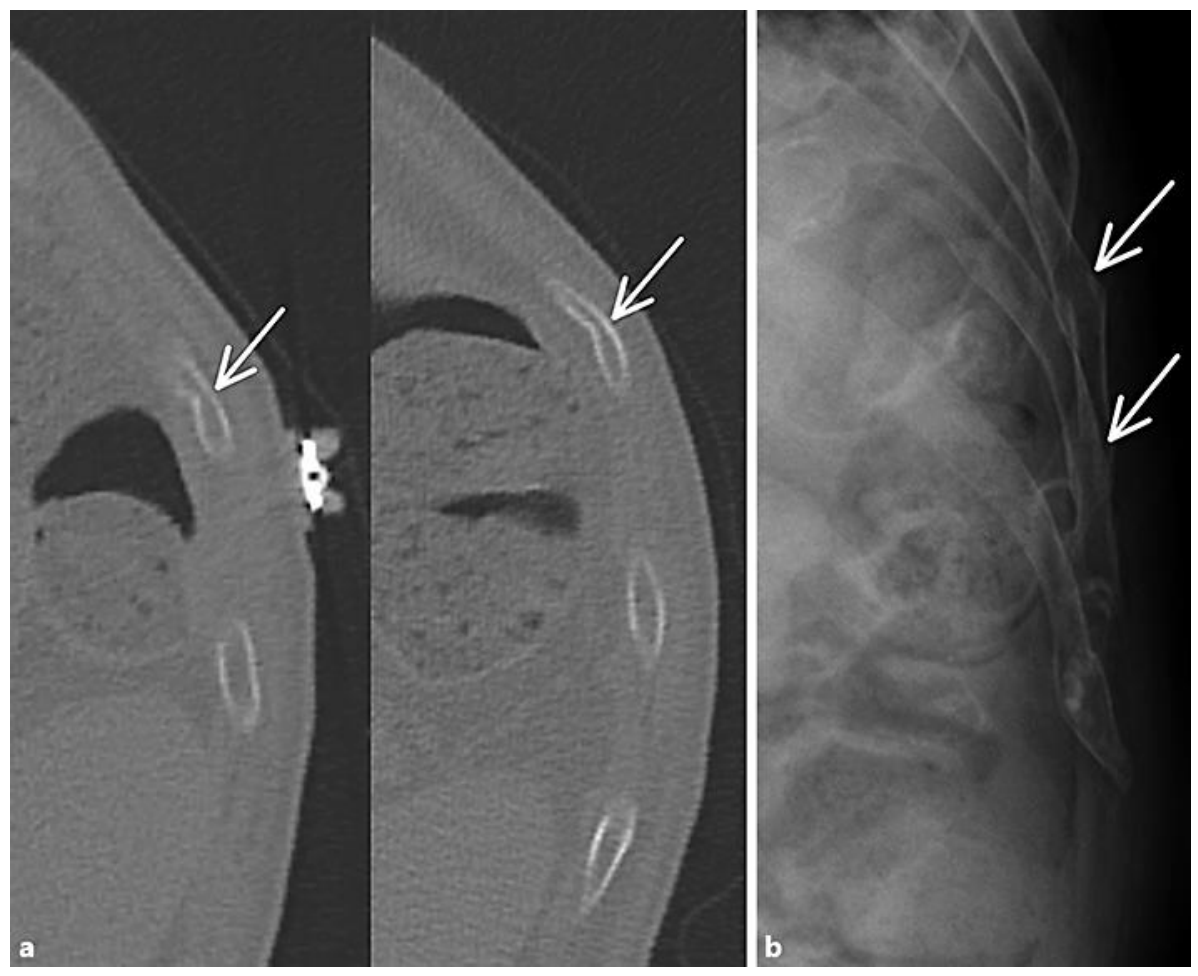

Fig. 2. Acute fractures of the 7th and 8th ventrolateral ribs on the left side on the day of stroke onset after cardiopulmonary resuscitation (a) and 5 days after treatment (b). Arrows mark the locations of the rib fractures. 